\title{
A virtual actuator approach for the fault tolerant control of unstable linear systems subject to actuator saturation and fault isolation delay
}

\author{
Damiano Rotondo ${ }^{1 \mathrm{a}}$, Jean-Christophe Ponsart ${ }^{\mathrm{b}}$, Didier Theilliol ${ }^{\mathrm{b}}$, Fatiha \\ Nejjaria ${ }^{\mathrm{a}}$, Vicenç Puig ${ }^{\mathrm{a}, \mathrm{c}}$ \\ ${ }^{a}$ Automatic Control Department, Universitat Politecnica de Catalunya (UPC) \\ Rambla de Sant Nebridi, 11, 08222 Terrassa, Spain \\ e-mails:damiano.rotondo@yahoo.it, fatiha.nejjari@upc.edu,vicenc.puig@upc.edu \\ ${ }^{b}$ Centre de Recherche en Automatique de Nancy (CRAN), Université de Lorraine \\ CNRS UMR 7039, 54506 Vandoeuvre-les-Nancy, France \\ e-mails: Jean-Christophe.Ponsart@univ-lorraine.fr, didier.theilliol@univ-lorraine.fr \\ ${ }^{c}$ Institut de Robotica i Informatica Industrial (IRI), UPC-CSIC \\ Carrer de Llorens i Artigas, 4-6, 08028 Barcelona, Spain
}

\begin{abstract}
This paper presents a fault tolerant control (FTC) strategy for unstable linear systems subject to actuator saturation and fault isolation delay. The solution relies on virtual actuators, an active fault-hiding method that reconfigures the faulty plant instead of the controller. The main contribution of the paper consists in the design of the virtual actuators with guarantees that, if at the fault isolation time the closed-loop system state is inside a region defined by a value of the Lyapunov function, the state trajectory will converge to zero despite the appearance of faults within a predefined set. In addition, the design of the nominal controller is performed so as to maximize the tolerated delay between the fault occurence and its isolation. Finally, the theoretical results are demonstrated using an example.
\end{abstract}

Keywords:

Actuator saturation, unstable open loop systems, linear matrix inequalities, active fault tolerant control system, virtual actuators.

\footnotetext{
${ }^{1}$ Corresponding author.
} 


\section{Introduction}

Real-world actuators are always subject to limits in the magnitude of the manipulated input. The control techniques that ignore these actuator limits can be affected by degraded performance, and may even lead to instability of the closed-loop system. Hence, recent research has focused on the analysis and synthesis of control systems with saturating actuators $[1,2]$. The developed solutions mainly use two approaches: the two-step paradigm, also called anti-windup compensation [3, 4], where a controller which does not explicitly take into account the saturation is designed, and then a compensator is added to handle the saturation constraints; and the one-step paradigm, also called direct control design [5,6], where the input constraints are taken into account at the controller design stage.

In recent years, fault tolerant control (FTC) techniques have been investigated, with the objective of maintaining the performances under fault occurrence close to the desired ones, and preserving stability conditions in the presence of faults $[7,8]$. Even though fault tolerance can be achieved straightforwardly through the so-called hardware redundancy, i.e. by adding redundant actuators and sensors that replace the faulty ones under fault occurrence, the analytical redundancy is often preferred in order to decrease the overall economic cost. The existing analytical redundancy approaches are usually classified into passive and active [9]. The passive FTC techniques are control laws that take into account the fault as a system perturbation. Thus, within certain margins, the control law has inherent fault tolerant capabilities, allowing the system to cope with the fault presence. On the other hand, the active FTC techniques compensate the faults either by selecting a precalculated control law or by synthesizing on-line a new control strategy. The adaptation of the control law is done by using some information about the fault so as to satisfy the control objectives with minimum performance degradation after the fault occurrence (see $[10,11]$ for a review).

Among the successful active FTC strategies, there is the fault-hiding paradigm [12], where the faulty plant is reconfigured instead of the controller. The advantage of this paradigm, with respect to other active FTC strategies, is that the property of fault tolerance can be added to an existing control scheme, without affecting the other properties, e.g. stability and performance, already attained by the controller under nominal situation. The controller is kept in the loop by inserting a reconfiguration block between the faulty plant and the controller when the fault occurs. The reconfiguration 
block is chosen so as to hide the fault from the controller point of view, allowing it to see the same plant as before the fault. In case of actuator faults, the reconfiguration block is named virtual actuator, because it generates a signal which has the same effect, or approximately the same, as the faulty actuator would have in the nominal system [13] (see Fig. 1 for a scheme illustrating the virtual actuator FTC concept). Initially proposed in a state-space formulation for LTI systems [13], this active FTC strategy has been successfully extended to linear parameter varying (LPV) [14], Takagi-Sugeno [15], piecewise affine [16], Lipschitz [17] and Hammerstein-Weiner [18] systems. An equivalent formulation in input-output form has been recently proposed in [19].

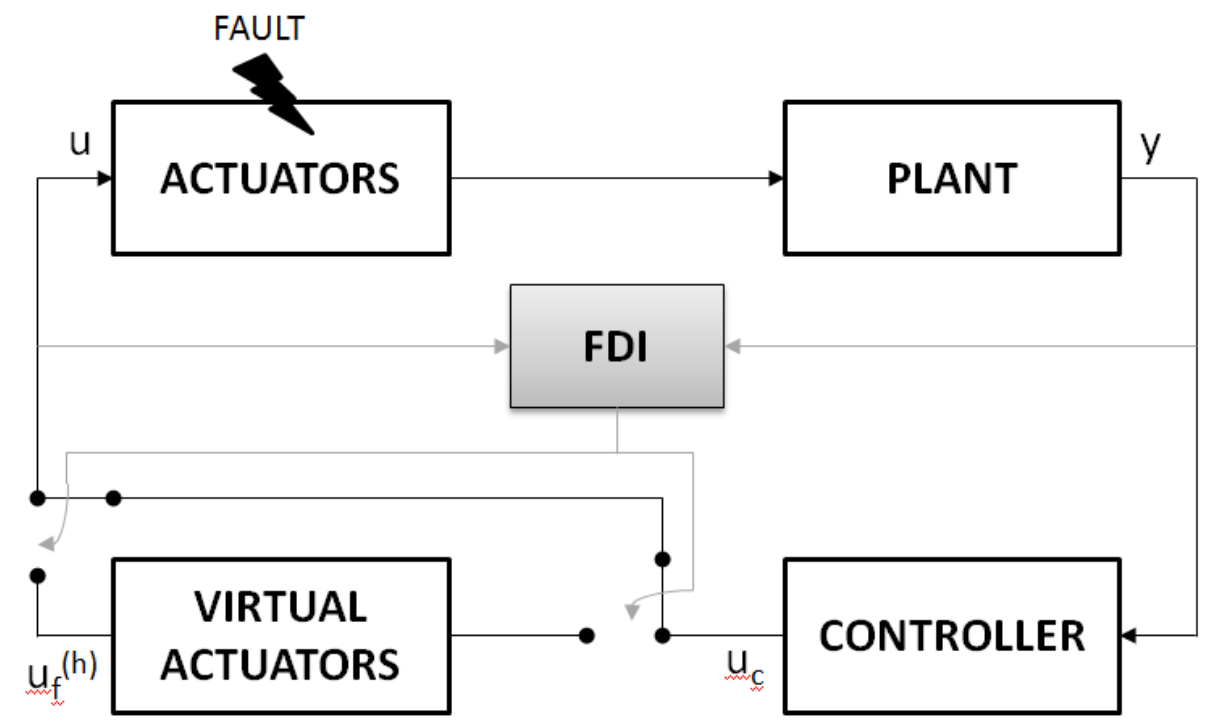

Figure 1: Virtual actuator FTC concept.

It is important to consider the actuator saturation constraints in the application of an FTC strategy, especially when actuator faults are considered. In fact, fault tolerance against actuator faults is usually achieved redistributing, in some way, the control effort corresponding to the faulty actuators among the remaining healthy ones. This redistribution may lead to saturation of both the faulty and the healthy actuators. Thus, if this fact is neglected in the FTC system design, severe performance degradation or instability may occur [20]. Some recent works have considered the problem 
of FTC systems subject to actuator saturations. [21] shows that failures resulting from loss of actuator effectiveness in systems with input saturations can be dealt with in the context of the absolute stability theory framework. [22] presents two kinds of fault tolerant controllers (fixed-gain and adaptive) for singular systems subject to actuator saturation. Both of these two controllers are in the form of a saturation avoidance feedback. [23] develops a fault tolerant control scheme that can achieve attitude tracking objective for a flexible spacecraft in the presence of partial loss of actuator effectiveness fault and actuator saturation using sliding mode control. The solution proposed by [24] avoids to use the failed control actuators in the event of a fault. Also, concepts such as graceful performance degradation [25, 26] and reference reconfiguration [27, 28] have been introduced in the context of FTC of systems subject to actuator saturations. However, only a few works have considered this problem for unstable systems. [29] has proposed a linear time varying (LTV) fault tolerant compensator, using the relevant ability of LTV compensators to achieve simultaneous stabilization of several systems. An active FTC scheme based on gain-scheduled $\mathcal{H}_{\infty}$ control and neural network for unstable systems has been proposed by [30]. Finally, [31] develops a robust fault tolerant scheme based on variable structure control for an orbiting spacecraft with a combination of unknown actuator failures and input saturation.

However, even though an active FTC system can react to faults more effectively than a passive FTC system can do, passive FTC techniques have been preferred to the active ones when dealing with unstable systems [29, $31,20]$. In fact, the active FTC strategies require a fault detection and isolation (FDI) module, and when unstable systems are considered, the time delay between the appearance of the fault and the moment in which the active strategy is activated (at the fault detection or isolation time) may destabilize the system. According to our knowledge, [30] is the only work dealing with active FTC for unstable system. However, in this reference, the issues arising from the FDI time delay were not considered. Also, another issue that has not been considered is the fact that, when dealing with unstable systems, the stability properties guaranteed by the control design are regional, i.e. hold only for inputs up to some size or for initial states inside a region of the state space [32]. The fault appearance, and the subsequent control system reconfiguration brought by the active FTC strategies change the regional stability properties of the control system, so it is necessary to take into account this fact explicitly when the system is subject to actuator 
saturations.

The main contribution of this paper consists in the design of an active FTC strategy for unstable systems subject to actuator saturation. Under the assumption that a nominal controller has been already designed using the direct control design paradigm to take into account the saturations, virtual actuators are added to the control loop for achieving fault tolerance against a predefined set of possible faults. In particular, faults affecting the actuators and causing a change in the system input matrix are considered. The design of the virtual actuators is performed in such a way that, if at the fault isolation time the closed-loop system state is inside a region defined by a value of the Lyapunov function, the state trajectory will converge to zero despite the appearance of the faults. Also, it is shown that it is possible to obtain some guarantees about the tolerated delay between the fault occurrence and its isolation. Moreover, the design of the nominal controller can be performed so as to maximize the tolerated delay.

It should be pointed out that, although saturations can be included within the Hammerstein-Weiner formulation of the virtual actuators, the approach proposed in this paper can be distinguished from the one introduced in [18] since less restrictive assumptions are required. In particular, some delay in the fault isolation is accepted, and the system matrix could be non-Hurwitz. In fact, although applicable to stable systems, the approach proposed hereafter focuses on the unstable ones.

The paper is structured as follows. Section 2 recalls some known results that will be used throughout the paper. Section 3 states the problems, that are solved in Section 4. The theoretical results are illustrated using an example in Section 5. Finally, the main conclusions are drawn in Section 6.

Notation: For a given matrix $M \in \mathbb{R}^{n_{r} \times n_{c}}$, the $i$ th row will be denoted as $M_{i}$, and the element located in its $i$ th row and $j$ th column as $M_{i, j}$. For brevity, symmetric elements in a matrix are denoted by $*$ and $M+M^{T}$ will be indicated as $H e\{M\}$. If a matrix $M \in \mathbb{R}^{n \times n}$ is symmetric, then $M \in \mathbb{S}^{n \times n}$. $I$ and $O$ denote the identity matrix and the zero matrix of appropriate dimensions, respectively. A matrix $M \in \mathbb{S}^{n \times n}$ is said positive definite $(M \succ 0)$ if all its eigenvalues are positive, and negative definite $(M \prec 0)$ if all its eigenvalues are negative. Moreover, the symbol $\otimes$ denotes the Kronecker product and $\dagger$ denotes the Moore-Penrose pseudoinverse. 


\section{Preliminaries}

Consider the autonomous nonlinear system

$$
\dot{x}(t)=f(x(t))
$$

where $x \in \mathbb{R}^{n_{x}}$ is the state and $f$ denotes a nonlinear function. For $x(0)=$ $x_{0} \in \mathbb{R}^{n_{x}}$, let us denote the state trajectory of the system (1) as $\psi\left(t, x_{0}\right)$. Then, the domain of attraction of the origin is

$$
\mathcal{S}:=\left\{x_{0} \in \mathbb{R}^{n_{x}}: \lim _{t \rightarrow+\infty} \psi\left(t, x_{0}\right)=0\right\}
$$

Let $P \succ O$ and denote

$$
\mathcal{E}(P, \rho)=\left\{x \in \mathbb{R}^{n_{x}}: x^{T} P x \leq \rho\right\}
$$

and let $V(x(t))=x(t)^{T} P x(t)$ be a candidate Lyapunov function. The ellipsoid $\mathcal{E}(P, \rho)$ is said to be contractively invariant if $\dot{V}(x(t))<0$ for all $x \in \mathcal{E}(P, \rho) \backslash\{0\}$. Clearly, if $\mathcal{E}(P, \rho)$ is contractively invariant, it is inside the domain of attraction $\mathcal{S}$ [33].

Remark 1. As stated in [34], there is a tradeoff between the degree of approximation of the domain of attraction and the simplicity of the representation. In the literature, several shapes for determining contractively invariant regions have been considered, e.g. polytopes, but ellipsoids are widely used due to their simplicity. For this reason, ellipsoids have been considered in this work, even though the general idea behind the developed theory could be adapted to more complex shapes, at the expense of increasing the complexity of the approach.

Now, let us consider the following linear time invariant (LTI) system subject to actuator saturations

$$
\begin{gathered}
\dot{x}(t)=A x(t)+B \operatorname{sat}(u(t)) \\
y(t)=C x(t)
\end{gathered}
$$

where $x \in \mathbb{R}^{n_{x}}$ is the state, $u \in \mathbb{R}^{n_{u}}$ is the control input, $y \in \mathbb{R}^{n_{y}}$ is the measured output, $A \in \mathbb{R}^{n_{x} \times n_{x}}$ is the state matrix, $B \in \mathbb{R}^{n_{x} \times n_{u}}$ is the input 
matrix, $C \in \mathbb{R}^{n_{y} \times n_{x}}$ is the output matrix, and sat $: \mathbb{R}^{n_{u}} \rightarrow \mathbb{R}^{n_{u}}$ is the saturation function, defined as

$$
\operatorname{sat}(u)=\left(\begin{array}{c}
\operatorname{sat}_{1}\left(u_{1}\right) \\
\vdots \\
\operatorname{sat}_{i}\left(u_{i}\right) \\
\vdots \\
\operatorname{sat}_{n_{u}}\left(u_{n_{u}}\right)
\end{array}\right), \quad \operatorname{sat}_{i}\left(u_{i}\right)=\left\{\begin{array}{cc}
\alpha_{i} & \left(u_{i}>\alpha_{i}\right) \\
u_{i} & \left(\left|u_{i}\right| \leq \alpha_{i}\right) \\
-\alpha_{i} & \left(u_{i}<-\alpha_{i}\right)
\end{array}\right.
$$

where $\alpha=\left(\alpha_{1}, \ldots, \alpha_{n_{u}}\right)^{T} \in \mathbb{R}^{n_{u}}$ is a given vector with positive entries. For an output feedback law $u(t)=g(y(t))=g(C x(t))$, let us define $\mathcal{L}(u, \alpha)$ the region of the state space in which the actuators are not saturated.

Then, the following lemma [35] gives the conditions for designing a dynamic output feedback controller for the system (4)-(5)

$$
\begin{aligned}
& \dot{x}_{c}(t)=A_{c} x_{c}(t)+B_{c} y(t) \\
& u_{c}(t)=C_{c} x_{c}(t)+D_{c} y(t)
\end{aligned}
$$

such that, if $u(t)=u_{c}(t)$, then $\mathcal{E}(P, 1) \subseteq \mathcal{S}$ and $\mathcal{E}(P, 1) \subseteq \mathcal{L}(u, \alpha)$, i.e. the controller will be such that for any initial closed-loop state vector satisfying

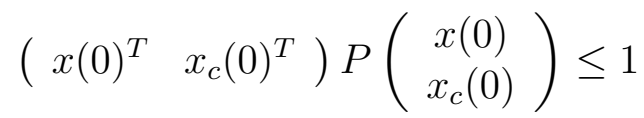

the control input never saturates, and the state trajectory converges to the origin.

Lemma 1. (Output Feedback Controller Design) Let $X, Y \in \mathbb{S}^{n_{x} \times n_{x}}, F \in$ $\mathbb{R}^{n_{x} \times n_{y}}, K \in \mathbb{R}^{n_{u} \times n_{x}}$ and $L \in \mathbb{R}^{n_{u} \times n_{y}}$ be such that

$$
\begin{gathered}
H e\{X A+F C\} \prec 0 \\
H e\{A Y+B K\} \prec 0 \\
\left(\begin{array}{ccc}
X & I & C^{T} L_{i}^{T} \\
I & Y & K_{i}^{T} \\
L_{i} C & K_{i} & \alpha_{i}^{2}
\end{array}\right) \succ 0 \quad\left(i=1, \ldots, n_{u}\right)
\end{gathered}
$$

then the controller (7)-(8), with matrices calculated as

$$
\left(\begin{array}{cc}
A_{c} & B_{c} \\
C_{c} & D_{c}
\end{array}\right)=\left(\begin{array}{cc}
Z & X B \\
0 & I
\end{array}\right)^{-1}\left(\begin{array}{cc}
-(A+B L C)^{T}-X A Y & F \\
K & L
\end{array}\right)\left(\begin{array}{cc}
-Y & 0 \\
C Y & I
\end{array}\right)^{-1}
$$




$$
Z=X-Y^{-1}
$$

is such that, for the closed-loop system obtained with $u(t)=u_{c}(t), \mathcal{E}(P, 1) \subseteq$ $\mathcal{S}$ and $\mathcal{E}(P, 1) \subseteq \mathcal{L}(u, \alpha)$ where

$$
P=\left(\begin{array}{cc}
X & Z \\
Z & Z
\end{array}\right)
$$

Proof. See [35].

Remark 2. The shape of the ellipsoidal invariant set can be fixed, or forced to be optimized in some desired sense, e.g. optimizing $\operatorname{det}(X)$ or $\operatorname{trace}(X)$, during the application of Lemma 1. However, this optimization could lead to an ellipsoid that favors some state variables more than the others, that may be undesired in some situations.

Let us recall the following lemma that relates a Lyapunov inequality with its dual version [36].

Lemma 2. (Equivalence of Lyapunov inequalities) Consider the following matrix inequality

$$
A^{T} Q+Q A \prec 0
$$

for a given matrix $A$ and $Q \succ 0$. Then, if (16) holds, also the following matrix inequality holds

$$
A Q^{-1}+Q^{-1} A^{T} \prec 0
$$

Proof. It is straightforward to obtain (17) by pre- and post-multiplying (16) by $Q^{-1}$.

Also, the following lemma gives a constraint on the scalar product of two vectors [37].

Lemma 3. (Magnitude of the scalar product of two vectors) Given two vectors $m$ and $x$, the existence of $Q \succ 0$ such that

$$
\left(\begin{array}{cc}
Q^{-1} & Q^{-1} m \\
m^{T} Q^{-1} & \gamma^{2}
\end{array}\right) \succ 0
$$

implies that $\left|m^{T} x\right| \leq \gamma \forall x \in \mathcal{E}(Q, 1)$. 
Proof. This lemma is a direct consequence of applying Schur complement to (18).

Finally, the last lemma provides some conditions for the decay or growth rate of a quadratic function of the state of an LTI system [38].

Lemma 4. (Exponential decay/growth of linear systems) Consider the following region of the complex plane

$$
\mathcal{D}=\left\{z \in \mathbb{C}: f_{\mathcal{D}}(z)=\chi+z \delta+\bar{z} \delta^{T}<0\right\}
$$

where $\chi \in \mathbb{S}^{n_{\mathcal{D}} \times n_{\mathcal{D}}}$ and $\delta \in \mathbb{R}^{n_{\mathcal{D}} \times n_{\mathcal{D}}}$, and the dynamical system

$$
\dot{x}(t)=A x(t)
$$

Assume that there exists $P \succ 0$ such that

$$
M_{\mathcal{D}}(A, P)=\chi \otimes P+\delta \otimes P A+\delta^{T} \otimes A^{T} P \prec 0
$$

Then, the quadratic function $V(x(t))=x(t)^{T} P x(t)$ satisfies

$$
\frac{1}{2} \frac{\dot{V}(x(t))}{V(x(t))} \in \mathcal{D} \cap \mathbb{R}
$$

for all $x(t) \neq 0$.

Proof. See [38].

\section{Problem Statement}

Let us consider the following system subject to actuator saturations

$$
\begin{gathered}
\dot{x}(t)=A x(t)+\mathfrak{B}(t) \operatorname{sat}(u(t)) \\
y(t)=C x(t)
\end{gathered}
$$

with

$$
\mathfrak{B}(t)=\left\{\begin{array}{cc}
B & t<t_{f} \\
B_{f} \in\left\{B_{f}^{(1)}, \ldots, B_{f}^{\left(n_{f}\right)}\right\} & t \geq t_{f}
\end{array}\right.
$$

where $B \in \mathbb{R}^{n_{x} \times n_{u}}$ and the corresponding LTI system obtained from (23), that corresponds to (4)-(5), will be referred to as nominal input matrix and 
nominal system, respectively, $B_{f} \in \mathbb{R}^{n_{x} \times n_{u}}$ and the corresponding LTI system obtained from (23) will be referred to as faulty input matrix and faulty system, respectively, $t_{f} \in \mathbb{R}^{+}$is the fault occurrence time and the function sat $(u(t))$ is defined as in (6). The $n_{f}$ matrices $B_{f}^{(1)}, \ldots, B_{f}^{\left(n_{f}\right)} \in \mathbb{R}^{n_{x} \times n_{u}}$ are such that

$$
\operatorname{rank}\left(B_{f}^{(h)}\right)<\operatorname{rank}(B)
$$

and the pairs

$$
\left(A, B_{f}^{(h)}\left(B_{f}^{(h)}\right)^{\dagger} B\right)
$$

are stabilizable, $\forall h=1, \ldots, n_{f}$.

Problem 1: Assume that an output feedback controller (7)-(8) has been designed for the nominal system using Lemma 1 , such that $\mathcal{E}(P, 1) \subseteq \mathcal{S}$ and $\mathcal{E}(P, 1) \subseteq \mathcal{L}(u, \alpha)$, and let us consider the control law

$$
u(t)=\left\{\begin{array}{cc}
u_{c}(t) & t<t_{I} \\
u_{f}^{(1)}(t) & t \geq t_{I}, \mathfrak{B}(t)=B_{f}^{(1)} \\
\vdots & \vdots \\
u_{f}^{\left(n_{f}\right)}(t) & t \geq t_{I}, \mathfrak{B}(t)=B_{f}^{\left(n_{f}\right)}
\end{array}\right.
$$

where $t_{I} \in \mathbb{R}^{+}, t_{I} \geq t_{f}$ is the fault isolation time, that is assumed to be provided by an FDI module. Design $u_{f}^{(1)}(t), \ldots, u_{f}^{\left(n_{f}\right)}(t)$ and maximize $\nu_{f} \in$ ]0,1] such that, for all $t \geq t_{I}, \mathcal{E}\left(P, \nu_{f}\right)$ is contractively invariant for the system (23)-(24) with control law (28), and $\mathcal{E}\left(P, \nu_{f}\right) \subseteq \mathcal{L}(u, \alpha)$.

In other words, in Problem 1 , it is wished to design $u_{f}^{(1)}(t), \ldots, u_{f}^{\left(n_{f}\right)}(t)$ and maximize the value of $\nu_{f}$ such that it is guaranteed that if at the fault isolation time $t_{I}$

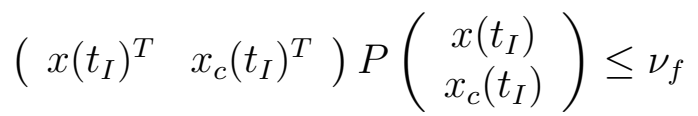

then the control input will not saturate for all $t \geq t_{I}$, and the state trajectory will converge to the origin.

It is clear that under the assumption of instantaneous fault isolation, if the closed-loop state trajectory has reached $\mathcal{E}\left(P, \nu_{f}\right)$, the solution of Problem 1 guarantees the state trajectory convergence under fault occurrence. However, 
this is not the case when there is a delay in the fault isolation, i.e. $t_{I}-t_{f}>$ 0. In fact, between the occurrence of the fault, that changes the system input matrix from $B$ to some $B_{f}^{(h)}$, and the fault isolation time, where the appropriate control $u_{f}^{(h)}$ begins to be applied, there is a time interval where the system is driven by the nominal control $u_{c}(t)$. During this period, there is no guarantee that, if the system had reached $\mathcal{E}\left(P, \nu_{f}\right)$ at time $t_{f}$, it will stay inside this region until $t_{I}$. This fact can lead to severe consequences, because if the state trajectory leaves $\mathcal{E}\left(P, \nu_{f}\right)$ before $t_{I}$, the system could be destabilized [30].

Given $\left[\begin{array}{ll}x\left(t_{f}\right)^{T} & x_{c}\left(t_{f}\right)^{T}\end{array}\right]^{T} \in \mathcal{E}\left(P, \nu_{f}\right)$, let us define, for the faulty system (23)-(24) with $\mathfrak{B}(t)=B_{f}^{(h)}, h=1, \ldots, n_{f}$, under control law $u(t)=u_{c}(t)$, the critical fault isolation time $\bar{t}_{I}^{(h)}\left(x\left(t_{f}\right), x_{c}\left(t_{f}\right)\right) \geq t_{f}$ as the time instant such that

$$
\left[x\left(\hat{t}_{I}^{(h)}\right)^{T} x_{c}\left(\hat{t}_{I}^{(h)}\right)^{T}\right]^{T} \in \mathcal{E}\left(P, \nu_{f}\right)
$$

but

$$
\left[x\left(\hat{t}_{I}^{(h)}+t_{\varepsilon}\right)^{T} \quad x_{c}\left(\hat{t}_{I}^{(h)}+t_{\varepsilon}\right)^{T}\right]^{T} \notin \mathcal{E}\left(P, \nu_{f}\right)
$$

for all $t_{\varepsilon}>0$.

Hence, it is interesting to solve the following problem, that improves the overall system robustness against the time isolation delay.

Problem 2: Find, among the output feedback controllers (7)-(8) that can be obtained from Lemma 1, the one that maximizes $\min _{h=1, \ldots, n_{f}} \hat{t}_{I}^{(h)}\left(x\left(t_{f}\right), x_{c}\left(t_{f}\right)\right)$ for all $\left[\begin{array}{ll}x\left(t_{f}\right)^{T} & x_{c}\left(t_{f}\right)^{T}\end{array}\right]^{T} \in \mathcal{E}\left(P, \nu_{f}\right)$, where $\hat{t}_{I}^{(h)}\left(x\left(t_{f}\right), x_{c}\left(t_{f}\right)\right)$ is an estimation of $\bar{t}_{I}^{(h)}\left(x\left(t_{f}\right), x_{c}\left(t_{f}\right)\right)$.

Remark 3. The critical fault isolation time indicates that the guarantees of non-saturating control input and state trajectory convergence to the origin given by the solution of Problem 1 are lost if $t_{I}>\hat{t}_{I}^{(h)}\left(x\left(t_{f}\right), x_{c}\left(t_{f}\right)\right)$. It is worth highlighting that the conditions given in this paper are sufficient, as always happens when using Lyapunov theory results. Hence, it is possible that the system exhibits state trajectory convergence to zero with non-saturating control input even if $t_{I}>\widehat{t}_{I}^{(h)}\left(x\left(t_{f}\right), x_{c}\left(t_{f}\right)\right)$. 


\section{Main Results}

\subsection{Solution to Problem 1}

The solution to Problem 1 proposed in this paper relies on the virtual actuator technique. The virtual actuators have the following structure

$$
\begin{gathered}
\dot{x}_{v}^{(h)}(t)=\left(A+B_{*}^{(h)} M^{(h)}\right) x_{v}^{(h)}(t)+\left(B-B_{*}^{(h)}\right) u_{c}(t), \quad x_{v}^{(h)}\left(t_{I}\right)=0 \\
u_{f}^{(h)}=N^{(h)}\left(u_{c}(t)-M^{(h)} x_{v}^{(h)}(t)\right)
\end{gathered}
$$

where $h=1, \ldots, n_{f}, x_{v}^{(h)}$ are the virtual actuators states, $M^{(h)} \in \mathbb{R}^{n_{u} \times n_{x}}$ are the virtual actuators gains to be designed, and the matrices $N^{(h)}$ and $B_{*}^{(h)}$ are given by

$$
\begin{gathered}
N^{(h)}=\left(B_{f}^{(h)}\right)^{\dagger} B \\
B_{*}^{(h)}=B_{f}^{(h)} N^{(h)}=B_{f}^{(h)}\left(B_{f}^{(h)}\right)^{\dagger} B
\end{gathered}
$$

Also, in order to obtain the fault-hiding characteristic, the output equation (24) is slightly changed after $t_{I}$, as follows

$$
y(t)=C\left(x(t)+x_{v}^{(h)}(t)\right) \quad t \geq t_{I}, \mathfrak{B}(t)=B_{f}^{(h)}
$$

The overall fault tolerant control scheme, made up by the system (23) with output equation (34) and control law (28), the output feedback controller (7)-(8) and the virtual actuators (30)-(31), is shown in Fig. 2.

Then, the following theorem provides the conditions to design the virtual actuators with guarantees that, if at the fault isolation time $t_{I}$, the closedloop system state is inside $\mathcal{E}\left(P, \nu_{f}\right)$, the state trajectory will converge to zero despite the change of the input matrix from $B$ to $B_{f}^{(h)}$ due to the fault.

Theorem 1. Let $X_{v a}^{-1} \in \mathbb{S}^{n_{x} \times n_{x}}$ and $\Gamma^{(h)} \in \mathbb{R}^{n_{u} \times n_{x}}, h=1, \ldots, n_{f}$ be such that

$$
\begin{gathered}
H e\left\{\left(\begin{array}{cc}
\nu_{f} A_{c l} P^{-1} & O_{2 n_{x} \times n_{x}} \\
\nu_{f} A_{*}^{(h)} P^{-1} & A X_{v a}^{-1}+B_{*}^{(h)} \Gamma^{(h)}
\end{array}\right)\right\} \prec 0 \\
\left(\begin{array}{cc}
X_{v a}^{-1} & \left(\Gamma_{j}^{(h)}\right)^{T} \\
\Gamma_{j}^{(h)} & \frac{\left(\frac{\alpha_{i}}{\left\|N_{i}^{(h)}\right\|}-\mu_{f}\right)^{2}}{n_{\tilde{u}}^{(h)}}
\end{array}\right) \succ 0 \quad i=1, \ldots, n_{u} \quad\left\|N_{i}^{(h)}\right\| \neq 0
\end{gathered}
$$




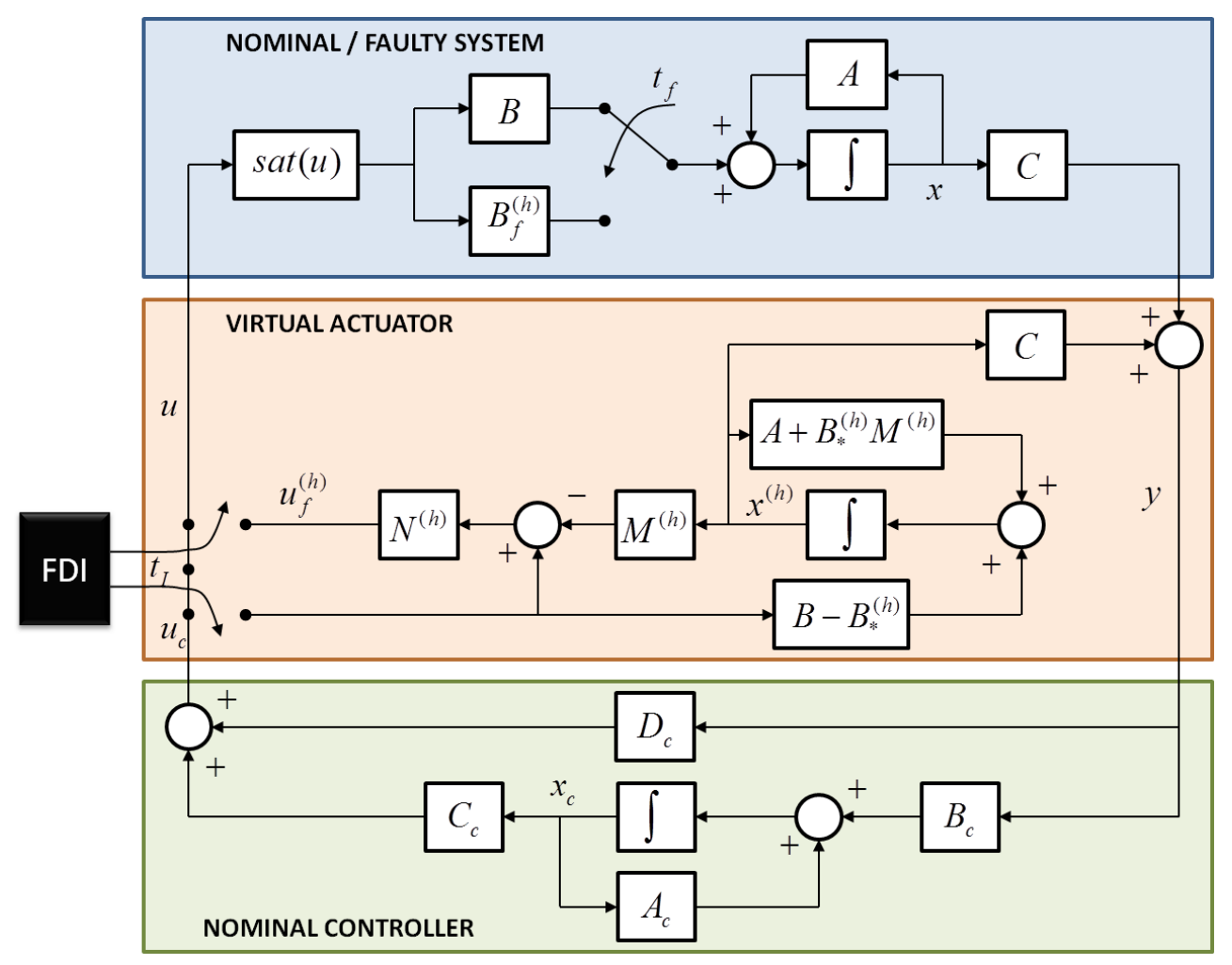

Figure 2: Overall fault tolerant control scheme. 
hold, where

$$
\begin{aligned}
& A_{c l}=\left(\begin{array}{cc}
A+B D_{c} C & B C_{c} \\
B_{c} C & A_{c}
\end{array}\right)
\end{aligned}
$$

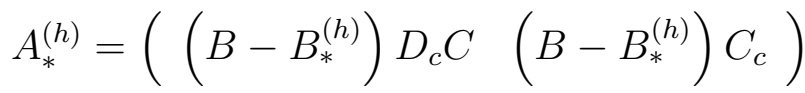

$$
\begin{aligned}
& \mu_{f}=\max _{\mathcal{E}\left(P, \nu_{f}\right)}\left\|u_{c}\right\|
\end{aligned}
$$

$n_{\tilde{u}}^{(h)}$ is the number of non-zero elements in $N_{i}^{(h)}$, and $j$ in (36) takes values corresponding to the indices of the non-zero elements in $N_{i}^{(h)}$. Then, if the virtual actuators gains $M^{(h)}$ in (30)-(31) are calculated as $M^{(h)}=\Gamma^{(h)} X_{v a}$, $\mathcal{E}\left(P, \nu_{f}\right)$ is contractively invariant for the system (23)-(24) with control law $(28)$, and $\mathcal{E}\left(P, \nu_{f}\right) \subseteq \mathcal{L}(u, \alpha), \forall t \geq t_{I}$.

Proof. First of all, let us notice that inside $\mathcal{L}(u, \alpha)$ the overall closed-loop system made up by the system (23), with output equation (34) and control law (28), the nominal controller (7)-(8), and the virtual actuator (30)-(31) can be brought to a block triangular form by means of a similarity transformation, by considering the state $x_{w}^{(h)}(t)=x(t)+x_{v}^{(h)}(t)$ instead of $x(t)$, and taking into account (33), as follows

$$
\left(\begin{array}{c}
\dot{x}_{c l}^{(h)}(t) \\
\dot{x}_{v}^{(h)}(t)
\end{array}\right)=\left(\begin{array}{cc}
A_{c l} & O_{2 n_{x} \times n_{x}} \\
A_{*}^{(h)} & A_{v}^{(h)}
\end{array}\right)\left(\begin{array}{c}
x_{c l}^{(h)}(t) \\
x_{v}^{(h)}(t)
\end{array}\right)
$$

where

$$
\begin{gathered}
x_{c l}^{(h)}(t)=\left(\begin{array}{c}
x_{w}^{(h)}(t) \\
x_{c}(t)
\end{array}\right) \\
A_{v}^{(h)}=A+B_{*}^{(h)} M
\end{gathered}
$$

Let us consider the following Lyapunov function to assess the stability of (40)

$$
V_{2}(t)=\left(\begin{array}{c}
x_{c l}^{(h)}(t) \\
x_{v}^{(h)}(t)
\end{array}\right)^{T}\left(\begin{array}{cc}
\frac{P}{\nu_{f}} & O_{2 n_{x} \times n_{x}} \\
O_{n_{x} \times 2 n_{x}} & X_{v a}
\end{array}\right)\left(\begin{array}{c}
x_{c l}^{(h)}(t) \\
x_{v}^{(h)}(t)
\end{array}\right)
$$

with $X_{v a} \succ 0$, that leads to the following Lyapunov inequality

$$
H e\left\{\left(\begin{array}{cc}
\frac{P}{\nu_{f}} & O_{2 n_{x} \times n_{x}} \\
O_{n_{x} \times 2 n_{x}} & X_{v a}
\end{array}\right)\left(\begin{array}{cc}
A_{c l} & O_{2 n_{x} \times n_{x}} \\
A_{*}^{(h)} & A_{v}^{(h)}
\end{array}\right)\right\} \prec 0
$$


By applying Lemma 2, (44) is equivalent to

$$
H e\left\{\left(\begin{array}{cc}
A_{c l} & O_{2 n_{x} \times n_{x}} \\
A_{*}^{(h)} & A_{v}^{(h)}
\end{array}\right)\left(\begin{array}{cc}
\nu_{f} P^{-1} & O_{2 n_{x} \times n_{x}} \\
O_{n_{x} \times 2 n_{x}} & X_{v a}^{-1}
\end{array}\right)\right\} \prec 0
$$

that can be brought to the LMI form (35) through the change of variable $\Gamma^{(h)}=M^{(h)} X_{v a}^{-1}$.

Hence, if (35) holds, the closed-loop trajectories of (40) will converge to the origin as long as the inputs $u$ do not saturate. The remaining part of the proof will aim at demonstrating that, thanks to the LMIs (36), if

$$
\left(x\left(t_{I}\right)^{T} \quad x_{c}\left(t_{I}\right)^{T}\right)^{T} \in \mathcal{E}\left(P, \nu_{f}\right)
$$

then the control inputs $u$ will not saturate, despite the additional effort brought by the virtual actuator.

To do so, let us notice that (31) is equivalent to

$$
u_{f, i}^{(h)}=N_{i}^{(h)}\left(u_{c}(t)-M^{(h)} x_{v}^{(h)}(t)\right)
$$

where $u_{f, i}^{(h)}$ refers to the $i$ th control input $\left(i=1, \ldots, n_{u}\right)$, such that the nonsaturating condition would be

$$
\begin{aligned}
\left|u_{f, i}^{(h)}\right| & =\left|N_{i}^{(h)}\left(u_{c}(t)-M^{(h)} x_{v}^{(h)}(t)\right)\right| \\
& \leq\left\|\begin{array}{l}
N_{i}^{(h)} \\
N_{i}^{(h)}
\end{array}\right\|\left(\begin{array}{l}
\left.\left\|u_{c}(t)\right\|+\left\|M^{(h)} x_{v}^{(h)}(t)\right\|\right) \\
\left.\mu_{f}+\left\|M^{(h)} x_{v}^{(h)}(t)\right\|\right) \leq \alpha_{i}
\end{array}\right.
\end{aligned}
$$

where $\mu_{f}$ is defined in (39) as

$$
\mu_{f}=\max _{\mathcal{E}\left(P, \nu_{f}\right)}\left\|u_{c}\right\|
$$

Hence, the following condition can be obtained from (48) for assuring the non-saturation of the control inputs

$$
\left\|N_{i}^{(h)}\right\|\left(\mu_{f}+\left\|M^{(h)} x_{v}^{(h)}(t)\right\|\right) \leq \alpha_{i}
$$

Notice that (49) is satisfied without need of additional considerations for $i$ such that $\left\|N_{i}^{(h)}\right\|=0$. In cases where $\left\|N_{i}^{(h)}\right\| \neq 0,(49)$ is equivalent to

$$
\left\|M^{(h)} x_{v}^{(h)}\right\| \leq \frac{\alpha_{i}}{\left\|N_{i}^{(h)}\right\|}-\mu_{f}
$$


The left-hand side of (50) is the norm of a vector. At the expense of introducing some conservativeness, it is possible to transform (50) into another condition concerning the norms of scalars. To do so, let us notice that, looking at (47), only the rows of $M^{(h)}$ corresponding to non-zero elements of $N_{i}^{(h)}$ will contribute to $u_{f, i}^{(h)}$. Let us denote these rows as $M_{j}^{(h)}$, and the number of non-zero elements of $N_{i}^{(h)}$ as $n_{\tilde{u}}^{(h)}$. Eq. (50) can be replaced with

$$
\sqrt{\sum_{j=1, N_{i, j}^{(h)} \neq 0}^{n_{u}}\left|M_{j}^{(h)} x_{v}^{(h)}\right|^{2}} \leq \frac{\alpha_{i}}{\left\|N_{i}^{(h)}\right\|}-\mu_{f}
$$

and notice that (51) holds if

$$
\left|M_{j}^{(h)} x_{v}^{(h)}\right| \leq \frac{\left(\frac{\alpha_{i}}{\left\|N_{i}^{(h)}\right\|}-\mu_{f}\right)}{\sqrt{n_{\tilde{u}}^{(h)}}} \quad j=1, \ldots, n_{u} \quad N_{i, j}^{(h)} \neq 0
$$

Applying Lemma 3 to (52), it is obtained that the existence of $Q \succ 0$ such that

$$
\left(\begin{array}{cc}
Q^{-1} & Q^{-1}\left(M_{j}^{(h)}\right)^{T} \\
M_{j}^{(h)} Q^{-1} & \frac{\left(\frac{\alpha_{i}}{\left\|N_{i}^{(h)}\right\|_{f}}\right)^{2}}{n_{\tilde{u}}^{(h)}}
\end{array}\right) \succ 0 \quad j=1, \ldots, n_{u} \quad N_{i, j}^{(h)} \neq 0
$$

implies that (52) holds $\forall x_{v}^{(h)} \in \mathcal{E}(Q, 1)$. By choosing $Q=X_{v a}$, and applying the change of variable $\Gamma^{(h)}=M^{(h)} X_{v a}^{-1}$ the LMIs (36) are obtained.

To complete the proof, it is needed to demonstrate that if (46) is true, then $x_{v}^{(h)}(t) \in \mathcal{E}\left(X_{v a}, 1\right) \forall t \geq t_{I}$. This is straightforward, since (46) corresponds to (29), that is equivalent to

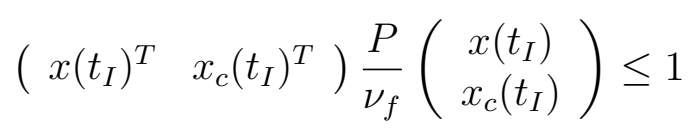

and, since $x_{v}^{(h)}\left(t_{I}\right)=0$ (see Eq. (30)), $x\left(t_{I}\right)$ in (54) can be replaced with $x_{w}^{(h)}\left(t_{I}\right)$, thus obtaining that $V_{2}\left(t_{I}\right) \leq 1$, where $V_{2}(t)$ is defined in (43). Due to the fact that $\dot{V}_{2}(t)<0 \forall t \geq t_{I}$, it follows that $x_{v}^{(h)}(t) \in \mathcal{E}\left(X_{v a}, 1\right)$. This completes the proof. 
Under the assumption of stabilizability of the pairs $\left(A, B_{*}^{(h)}\right)$, the feasibility of the conditions (35)-(36) provided by Theorem 1 depends on the value of $\nu_{f}$. The smaller is $\nu_{f}$, the more likely is the feasibility of (35)-(36).

The definition of Problem 1 involves the maximization of $\nu_{f}$ subject to the feasibility of (35)-(36). This problem can be solved applying any gradientfree constrained optimization algorithm [39].

Remark 4. Notice that, due to the block-triangularity of the state matrix in (40), a necessary condition for the closed-loop system stability is that $A_{v}^{(h)}$, defined in (42) is stable. The existence of $M$ such that $A_{v}^{(h)}$ is stable is guaranteed by the fact that the pairs $\left(A, B_{*}^{(h)}\right)$ are stabilizable (see $\left.(27)\right)$.

Remark 5. Solving Problem 1 using Theorem 1 involves finding $\mu_{f}$ defined in (39). $\mu_{f}$ can be found using optimization algorithms, e.g. the fmincon function in the Matlab Optimization Toolbox [40]. Due to the linearity of the control input $u_{c}$ with respect to the states $x$ and $x_{c}$ (see Eq. (8)), it is possible to reduce the inequality constraint given by $\mathcal{E}\left(P, \nu_{f}\right)$ to an equality constraint, by searching the maximum of $u_{c}$ on the frontier of $\mathcal{E}\left(P, \nu_{f}\right)$.

\subsection{Solution to Problem 2}

As a first step to solve Problem 2, let us consider the following theorem, that provides $\hat{t}_{I}^{(h)}\left(x\left(t_{f}\right), x_{c}\left(t_{f}\right)\right)$ for all $\left[x\left(t_{f}\right)^{T} \quad x_{c}\left(t_{f}\right)^{T}\right]^{T} \in \mathcal{E}\left(P, \nu_{f}\right)$.

Theorem 2. Let $\lambda^{(h)} \in \mathbb{R}^{+}$be such that

$$
-2 \lambda^{(h)} P+H e\left\{P\left(\begin{array}{cc}
A+B_{f}^{(h)} D_{c} C & B_{f}^{(h)} C_{c} \\
B_{c} C & A_{c}
\end{array}\right)\right\} \prec 0
$$

and let $\left[x\left(t_{f}\right)^{T} \quad x_{c}\left(t_{f}\right)^{T}\right]^{T}=x_{f} \in \mathcal{E}\left(P, \nu_{f}\right)$. Then

$$
\left[x(t)^{T} \quad x_{c}(t)^{T}\right]^{T} \in \mathcal{E}\left(P, \nu_{f}\right) \quad \forall t \in\left[t_{f}, \hat{t}_{I}^{(h)}\left(x\left(t_{f}\right), x_{c}\left(t_{f}\right)\right)\right]
$$

with

$$
\hat{t}_{I}^{(h)}\left(x\left(t_{f}\right), x_{c}\left(t_{f}\right)\right)=t_{f}+\frac{1}{2 \lambda^{(h)}} \ln \left(\frac{\nu_{f}}{x_{f}^{T} P x_{f}}\right)
$$


Proof. The faulty system (23)-(24), with $\mathfrak{B}(t)=B_{f}^{(h)}$, together with the output feedback controller (7)-(8), can be rewritten in the closed-loop autonomous form as

$$
\left(\begin{array}{c}
\dot{x}(t) \\
\dot{x}_{c}(t)
\end{array}\right)=\left(\begin{array}{cc}
A+B_{f}^{(h)} D_{c} C & B_{f}^{(h)} C_{c} \\
B_{c} C & A_{c}
\end{array}\right)\left(\begin{array}{c}
x(t) \\
x_{c}(t)
\end{array}\right)
$$

Let us apply Lemma 4 to (58) using the region $\operatorname{Re}(z)<\lambda^{(h)}$, that corresponds to (19) with $\chi=-2 \lambda^{(h)}$ and $\delta=1$, such that (21) reads as

$$
-2 \lambda^{(h)} P+H e\left\{P\left(\begin{array}{cc}
A+B_{f}^{(h)} D_{c} C & B_{f}^{(h)} C_{c} \\
B_{c} C & A_{c}
\end{array}\right)\right\} \prec 0
$$

Hence, if (59) holds, (22) is true for the quadratic function $V\left(x(t), x_{c}(t)\right)=$

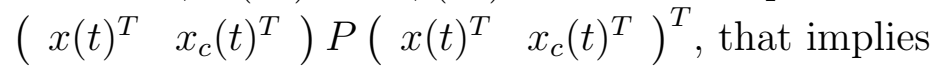

$$
V\left(x(t), x_{c}(t)\right) \leq V\left(x_{f}\right) e^{2 \lambda^{(h)}\left(t-t_{f}\right)}=x_{f}^{T} P x_{f} e^{2 \lambda^{(h)}\left(t-t_{f}\right)}
$$

By considering the condition $V\left(x(t), x_{c}(t)\right) \leq \nu_{f}$, that defines $\mathcal{E}\left(P, \nu_{f}\right)$, it is straightforward to obtain (56).

From (57) it can be seen that, to attain a solution to Problem 2, it is necessary to minimize $\lambda=\max _{h=1, \ldots, n_{f}} \lambda^{(h)}$. Hence, this solution is given by the following corollary, which is obtained combining Lemma 1 and Theorem 2.

Corollary 1. Let $X, Y \in \mathbb{S}^{n_{x} \times n_{x}}, F \in \mathbb{R}^{n_{x} \times n_{y}}, K \in \mathbb{R}^{n_{u} \times n_{x}}, L \in \mathbb{R}^{n_{u} \times n_{y}}$, $F^{(h)} \in \mathbb{R}^{n_{x} \times n_{y}}$ and $N^{(h)} \in \mathbb{R}^{n_{x} \times n_{x}}, h=1, \ldots, n_{f}$, correspond to the solution to the following constrained minimization problem

$$
\min \lambda
$$

subject to (10)-(12) and

$$
-2 \lambda\left(\begin{array}{cc}
X & I \\
I & Y
\end{array}\right)+H e\left\{\left(\begin{array}{cc}
X A+F^{(h)} C & N^{(h)} \\
A+B_{f}^{(h)} L C & A Y+B_{f}^{(h)} K
\end{array}\right)\right\} \prec 0 \quad \forall h=1, \ldots, n_{f}
$$

Then, the output feedback controller (7)-(8), with matrices calculated as (13)-(14) maximizes $\min _{h=1, \ldots, n_{f}} \hat{t}_{I}^{(h)}\left(x\left(t_{f}\right), x_{c}\left(t_{f}\right)\right)$ for all $\left[x\left(t_{f}\right)^{T} \quad x_{c}\left(t_{f}\right)^{T}\right]^{T} \in$ 
$\mathcal{E}\left(P, \nu_{f}\right)$, where $\hat{t}_{I}^{(h)}\left(x\left(t_{f}\right), x_{c}\left(t_{f}\right)\right)$ is the estimation of $\bar{t}_{I}^{(h)}\left(x\left(t_{f}\right), x_{c}\left(t_{f}\right)\right)$ obtained as (57) with $P$ defined as in (14)-(15)

$$
P=\left(\begin{array}{cc}
X & X-Y^{-1} \\
X-Y^{-1} & X-Y^{-1}
\end{array}\right)
$$

PROOF. The design condition (62) corresponds to the analysis condition (55) with $\lambda=\lambda^{(h)}$. In fact, by applying a congruent transformation to (55) with

$$
\Gamma=\left(\begin{array}{cc}
I & 0 \\
Y & -Y
\end{array}\right)
$$

and $\lambda=\lambda^{(h)}$, the following is obtained

$-2 \lambda\left(\begin{array}{cc}X & I \\ I & Y\end{array}\right)+H e\left\{\left(\begin{array}{cc}X A+X B_{f}^{(h)} C_{c} & \Upsilon^{(h)} \\ A+B_{f}^{(h)} D_{c} C & A Y+B_{f}^{(h)} D_{c} C Y-B_{f}^{(h)} C_{c} Y\end{array}\right)\right\}<0$

with

$$
\Upsilon^{(h)}=X A Y+X B_{f}^{(h)} D_{c} C Y-X B_{f}^{(h)} C_{c} Y+Z B_{c} C Y-Z A_{c} Y
$$

From (64), (62) can be obtained using the following change of variables

$$
\left(\begin{array}{cc}
N^{(h)} & F^{(h)} \\
K & L
\end{array}\right)=\left(\begin{array}{cc}
X A Y & 0 \\
0 & 0
\end{array}\right)+\left(\begin{array}{cc}
Z & X B_{f}^{(h)} \\
0 & I
\end{array}\right)\left(\begin{array}{cc}
A_{c} & B_{c} \\
C_{c} & D_{c}
\end{array}\right)\left(\begin{array}{cc}
-Y & 0 \\
C Y & I
\end{array}\right)
$$

Since a common $\lambda$ is being used, it is clear that $\lambda=\max _{h=1, \ldots, n_{f}} \lambda^{(h)}$, and by minimizing $\lambda$, we are maximizing $\min _{h=1, \ldots, n_{f}} \hat{t}_{I}^{(h)}$, defined as in (57).

Finally, in case that the design of an FTC system that solves Problem 1 and Problem 2 at the same time is desired, the following algorithm summarizes the necessary steps to do so

Find $X, Y, F, K, L, F^{(h)}, N^{(h)}, h=1, \ldots, n_{f}$ that minimize $\lambda$ subject to (10)-(12) and (62);

Calculate the controller matrices $A_{c}, B_{c}, C_{c}, D_{c}$ using (13)-(14);

Find $X_{v a}^{-1}, \Gamma^{(h)}, h=1, \ldots, n_{f}$ that maximize $\nu_{f}$ subject to (35)-(36);

Calculate the virtual actuator gain $M^{(h)}=\Gamma^{(h)} X_{v a}$.

Algorithm 1: Algorithm for solving Problem 1 and Problem 2. 


\section{Example}

Let us consider a system subject to actuator saturations as in (23)-(24), with

$$
\begin{aligned}
& A=\left(\begin{array}{cc}
2 & 0 \\
1 & 1.5
\end{array}\right) \quad C=\left(\begin{array}{ll}
1 & 0 \\
0 & 1
\end{array}\right) \\
& \mathfrak{B}(t)=\left\{\begin{array}{c}
B=\left(\begin{array}{ll}
1 & 0 \\
0 & 1
\end{array}\right) \quad t<t_{f} \\
B_{f}=\left(\begin{array}{ll}
1 & 0 \\
0 & 0
\end{array}\right) \quad t \geq t_{f}
\end{array}\right.
\end{aligned}
$$

and $\operatorname{sat}(u)$ defined as in (6) with $\alpha_{i}=10, i=1,2$. Notice that $\operatorname{rank}\left(B_{f}\right)=1$ and $\operatorname{rank}(B)=2$, i.e. (26) holds. Also, in this case, $B_{*}$ calculated as in (33) equals $B_{f}$, and it is easy to verify that the pair $\left(A, B_{*}\right)$ is stabilizable, since the corresponding controllability matrix has rank 2 .

By choosing $X=I$ in order to guarantee that, if $x_{c}(0)=0$, the control input will never saturate, and the state trajectory will converge to the origin if $x_{1}(0)^{2}+x_{2}(0)^{2} \leq 1$, Lemma 1 is applied, and the LMIs (10)-(12) are solved using the YALMIP toolbox [41] with SeDuMi solver [42]. Then, the controller matrices are calculated using (13), as follows

$$
\begin{array}{ccc}
A_{c}=\left(\begin{array}{cc}
-16.6325 & -0.2556 \\
-0.3130 & -15.9459
\end{array}\right) & B_{c}=\left(\begin{array}{cc}
-16.0585 & 0.1392 \\
-0.8227 & -15.6573
\end{array}\right) \\
C_{c}=\left(\begin{array}{cc}
0.0894 & 0.0255 \\
0.0218 & 0.0563
\end{array}\right) & D_{c}=\left(\begin{array}{cc}
-2.4119 & -0.3530 \\
-0.4528 & -1.6959
\end{array}\right)
\end{array}
$$

and the Lyapunov matrix $P$ is given by (15)

$$
P=\left(\begin{array}{cccc}
1 & 0 & 0.9108 & -0.0144 \\
0 & 1 & -0.0144 & 0.9471 \\
0.9108 & -0.0144 & 0.9108 & -0.0144 \\
-0.0144 & 0.9471 & -0.0144 & 0.9471
\end{array}\right)
$$

Problem 1, as described in Section 4.1, is solved applying an iterative optimization algorithm, obtaining a maximum value $\nu_{f}=0.02$, that corresponds to a value of $\mu_{f}$, defined as in (39), equal to 1.2486 .

Then, by applying Theorem 1 , the matrix $X_{v a}$ and the virtual actuator gain $M$ are given by

$$
X_{v a}=\left(\begin{array}{ll}
0.7495 & 1.4030 \\
1.4030 & 3.0214
\end{array}\right)
$$




$$
M=\left(\begin{array}{cc}
-6.3250 & -9.1499 \\
0 & 0
\end{array}\right)
$$

Let us consider a simulation that lasts $20 s$ with $x(0)=\left(\begin{array}{ll}1 & 0\end{array}\right)^{T}, x_{c}(0)=$ $\left(\begin{array}{ll}0 & 0\end{array}\right)^{T}$, and $t_{f}=2 \mathrm{~s}$. At first, the assumption of instantaneous fault isolation is done, i.e. $t_{I}=t_{f}$. Since $\left(x(0)^{T} \quad x_{c}(0)^{T}\right)^{T} \in \mathcal{E}(P, 1)$, the state trajectory will converge towards the origin and the control input will not saturate in the time interval $\left[0, t_{f}\right]$, as shown in Fig. 3 and Fig. 4, respectively.
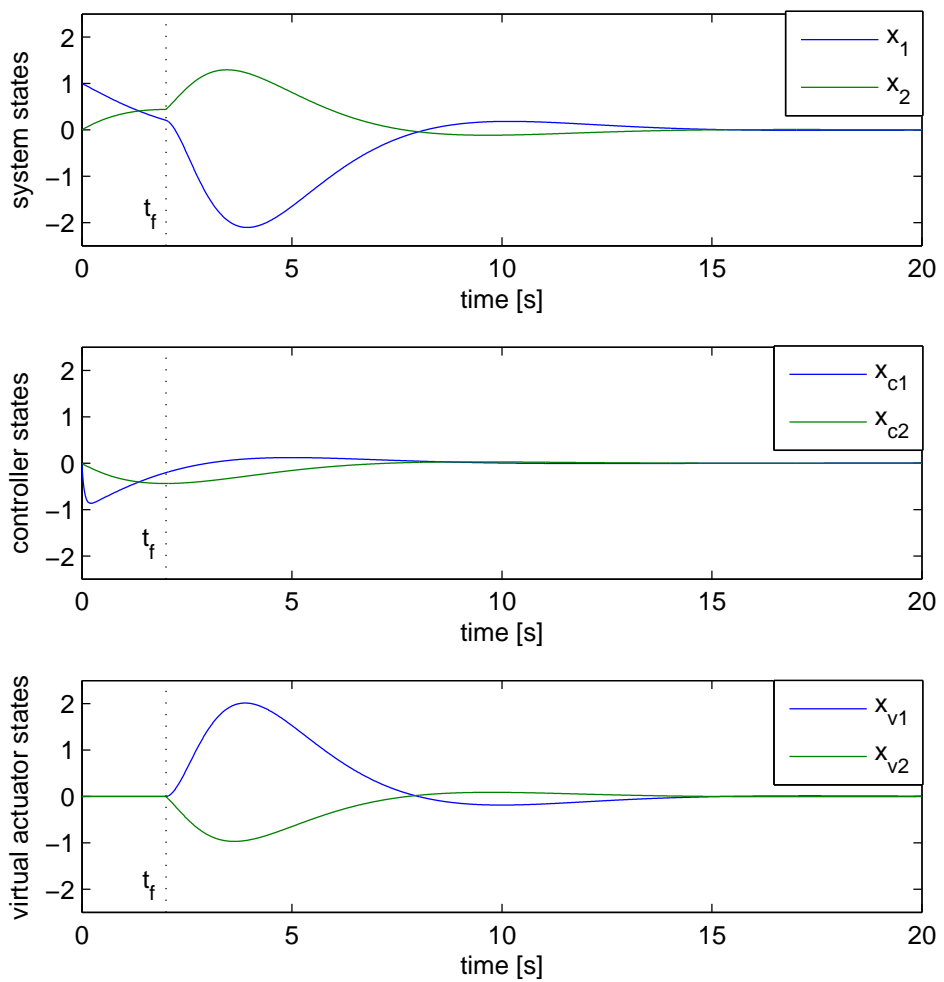

Figure 3: State trajectory, $t_{f}=t_{I}=2 s$.

Also, as shown in Fig. 5, the evolution of the Lyapunov function

$$
V(t)=\left(\begin{array}{c}
x_{c l}(t) \\
x_{v}(t)
\end{array}\right)^{T}\left(\begin{array}{cc}
P & O_{4 \times 2} \\
O_{2 \times 4} & \nu_{f} X_{v a}
\end{array}\right)\left(\begin{array}{c}
x_{c l}(t) \\
x_{v}(t)
\end{array}\right)
$$




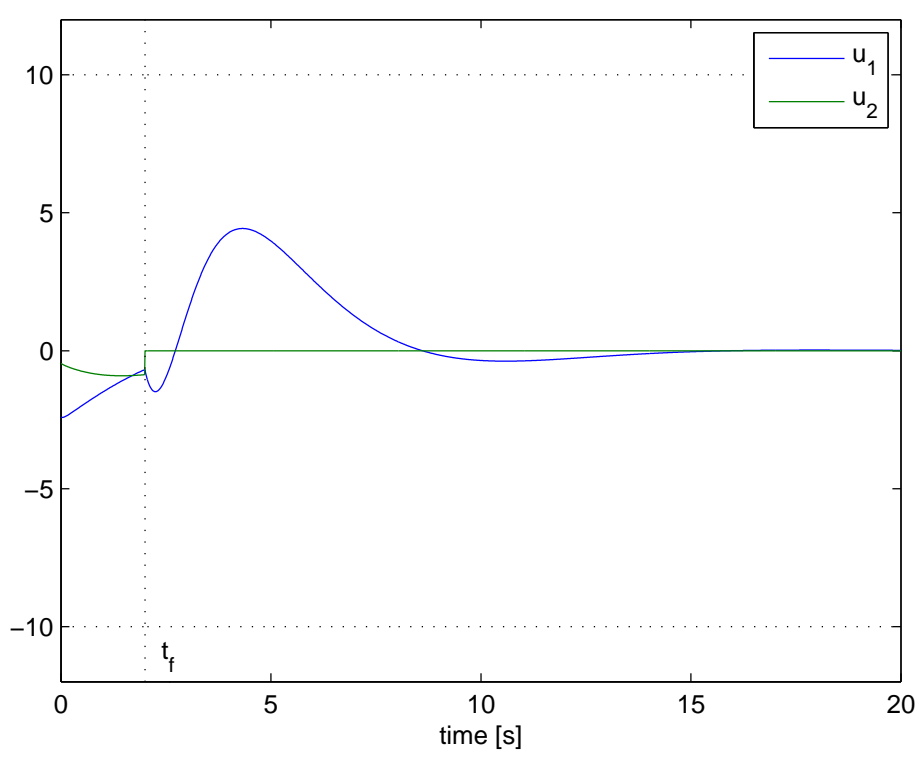

Figure 4: Control inputs, $t_{f}=t_{I}=2 s$.

is such that $V\left(t_{f}\right)=0.0166<\nu_{f}=0.02$. Hence, according to Theorem 1 , the activation of the virtual actuator at time $t_{I}=t_{f}$ guarantees that the system trajectory will converge to the origin with non-saturating control inputs despite the change in the input matrix from $B$ to $B_{f}$. This is shown in Fig. 3, where it can be seen clearly that, due to the activation of the virtual actuator, the states $x_{v 1}$ and $x_{v 2}$ take values different from zero, and in Fig. 4, where the reconfiguration of the control inputs brought by the change in the control law from $u_{c}(t)$ to $u_{f}(t)$ is depicted. Also, as expected, the Lyapunov function $V(t)$ takes decreasing-in-time values despite the fault occurrence, as shown in Fig. 5.

To conclude the analysis of the results, let us analyze the trajectories in the phase planes, shown in Figs. 6-8. It can be seen that the evolution of $x(t)$, $x_{c}(t)$ and $x_{v}(t)$ is such that at time $t_{I}$ all the states are inside $\mathcal{E}\left(P, \nu_{f}\right)$, whose projections in the considered phase plane are depicted in magenta color. After $t_{I}$, the state $x_{w}(t)=x(t)+x_{v}(t)$ continues smoothly the evolution of the state $x(t)$ before $t_{I}$; on the other hand, $x(t)$ will eventually converge to the origin because both $x_{w}(t)$ and $x_{v}(t)$ will do so.

Let us now consider the more realistic case where $t_{I}>t_{f}$, using the 

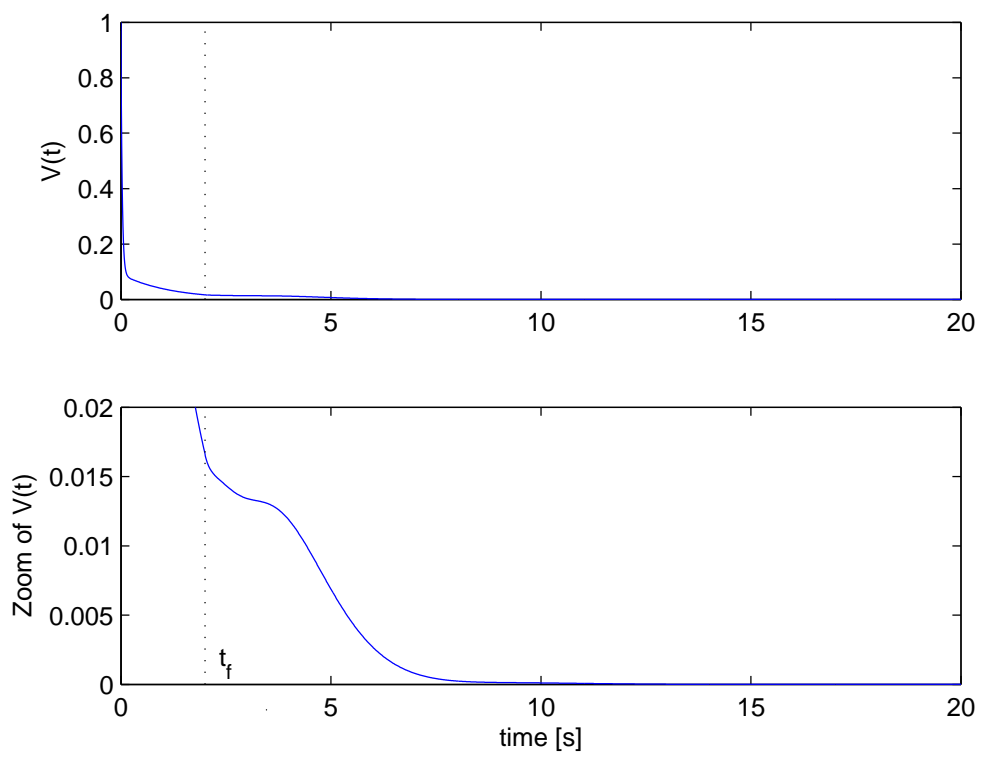

Figure 5: Lyapunov function $V(t), t_{f}=t_{I}=2 s$.

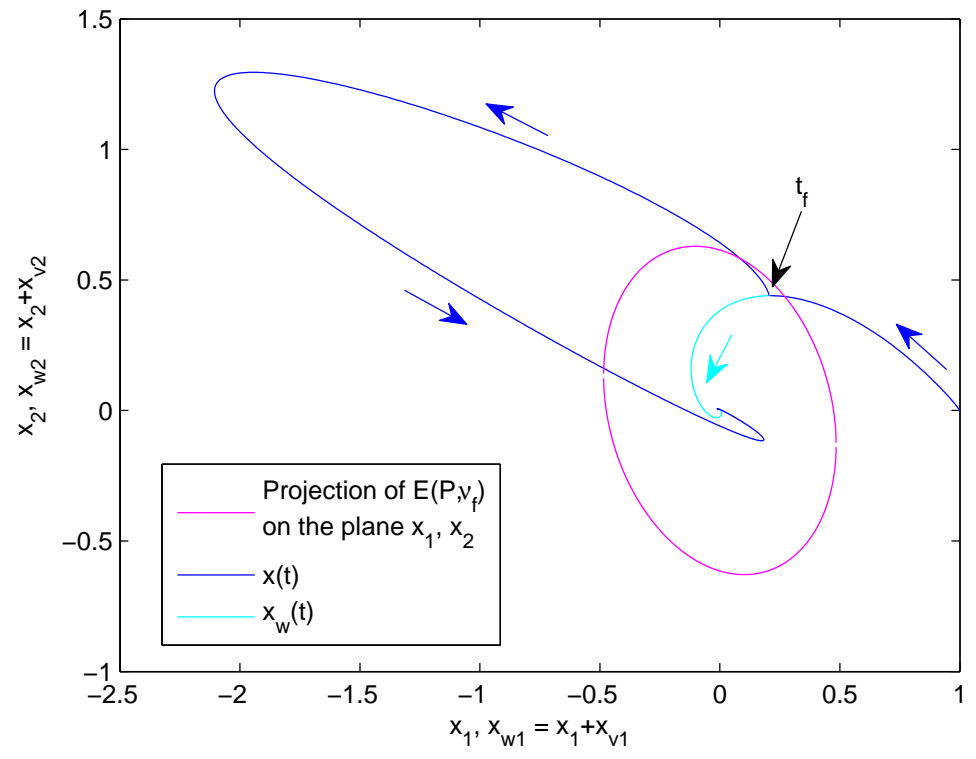

Figure 6: Phase plane of $x(t)$ and $x_{w}(t), t_{f}=t_{I}=2 s$. 


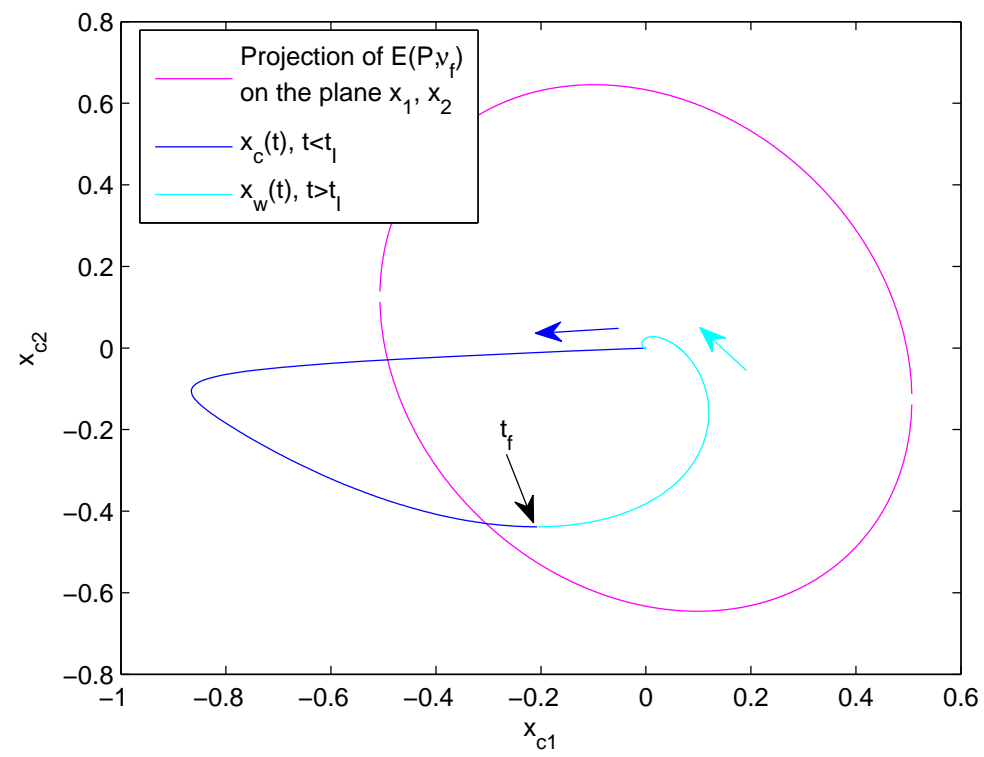

Figure 7: Phase plane of $x_{c}(t), t_{f}=t_{I}=2 s$.

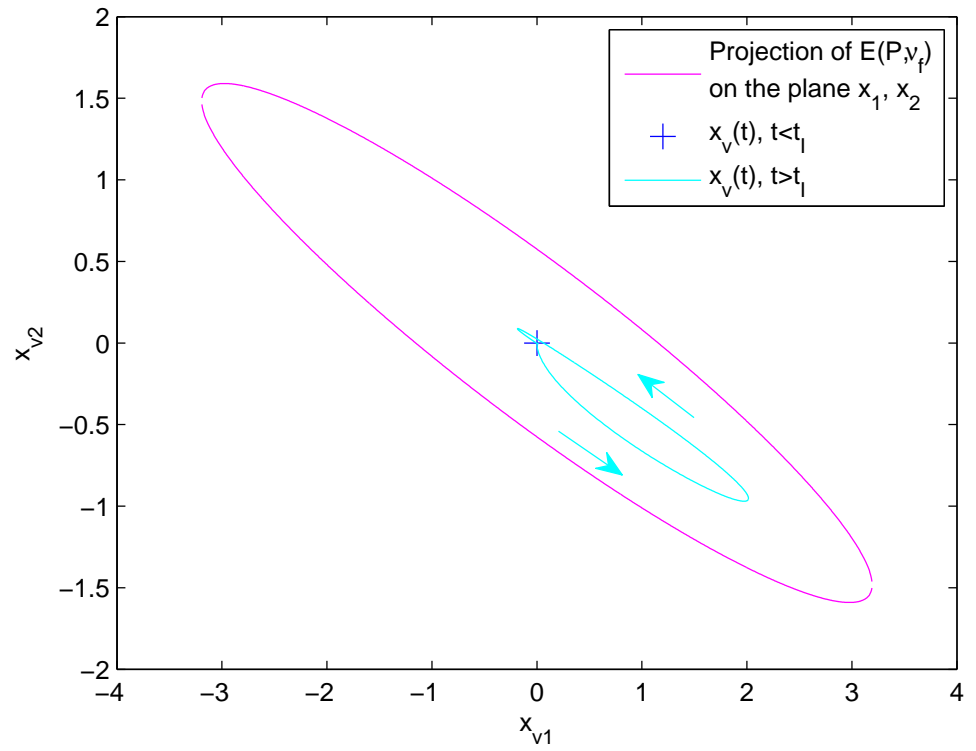

Figure 8: Phase plane of $x_{v}(t), t_{f}=t_{I}=2 s$. 
results obtained in Section 4.2. The application of Theorem 2 gives a value $\lambda=2.1873$, that corresponds to

$$
\hat{t}_{I}=t_{f}+\frac{1}{4.3746} \ln \left(\frac{0.02}{x_{f}^{T} P x_{f}}\right)
$$

At time $t_{f}=2 s, x_{f}^{T} P x_{f}=0.0166$, so that (68) gives $\hat{t}_{I}=2.043 \mathrm{~s}$, that is, if the fault isolation is performed within $0.043 \mathrm{~s}$, the system state is guaranteed to be inside $\mathcal{E}\left(P, \nu_{f}\right)$ for $\nu_{f}=0.02$ when the control $u_{f}(t)$ begins to be used instead of $u_{c}(t)$. This is confirmed by the simulation, as shown in Fig. 9. It is worth remarking that $\hat{t}_{I}$ is only an estimation of the critical fault isolation time that, for the considered example, can be determined by various simulations as $\bar{t}_{I}=2.088 \mathrm{~s}$.

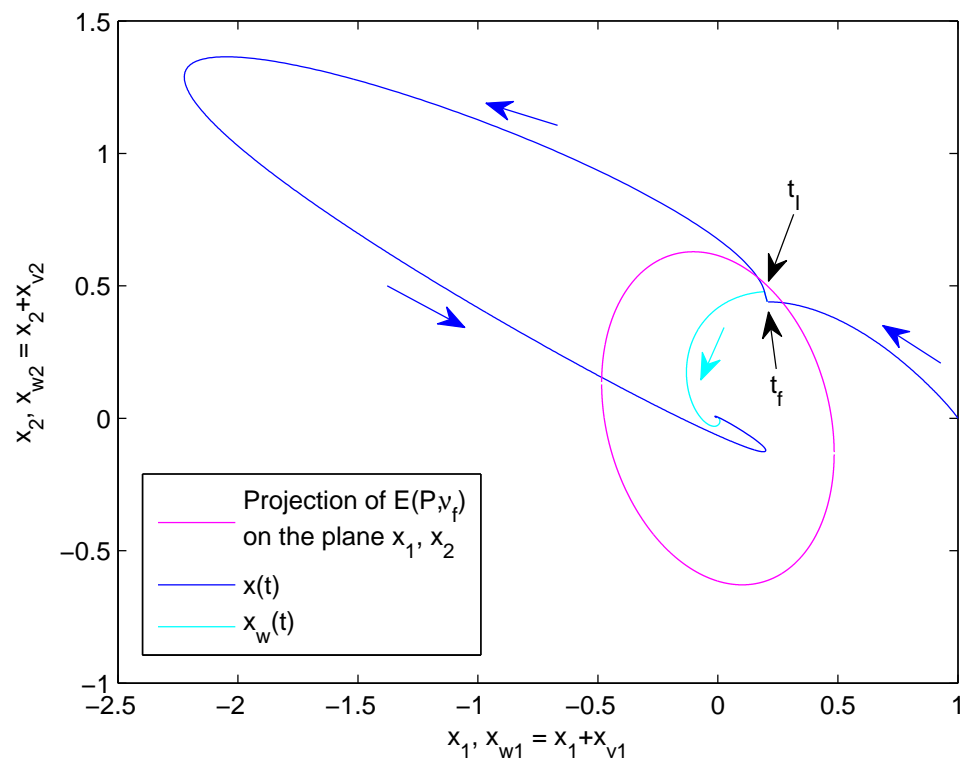

Figure 9: Phase plane of $x(t)$ and $x_{w}(t), t_{f}=2 s, t_{I}=2.043 s$.

By applying Corollary 1, a value $\lambda=0$ is achieved with the controller matrices

$$
A_{c}=\left(\begin{array}{ll}
-25.3686 & -27.1944 \\
-28.6509 & -75.3926
\end{array}\right) \quad B_{c}=\left(\begin{array}{cc}
-17.5185 & -8.4822 \\
-14.1644 & -39.2691
\end{array}\right)
$$




$$
C_{c}=\left(\begin{array}{cc}
0.0413 & 0.0199 \\
-0.0210 & -0.1043
\end{array}\right) \quad D_{c}=\left(\begin{array}{cc}
-3.7867 & -4.1372 \\
-1.6709 & -4.1821
\end{array}\right)
$$

and Lyapunov matrix

$$
P=\left(\begin{array}{cccc}
1 & 0 & 0.8191 & -0.2823 \\
0 & 1 & -0.2823 & 0.3018 \\
0.8191 & -0.2823 & 0.8191 & -0.2823 \\
-0.2823 & 0.3018 & -0.2823 & 0.3018
\end{array}\right)
$$

Notice that achieving the case $\lambda=0$, that would correspond to $\hat{t}_{I}=\infty$ using (57), is equivalent to the existence of a nominal controller that is robust against the considered fault. In fact, by repeating the simulation with this controller, assuming that the fault is not isolated during the simulation, it can be seen that the state trajectory with the nominal controller will still converge to zero despite the fault occurrence (see blue line in Fig. 10). On the other hand, the closed-loop system with the controller that had been designed without applying Corollary 1, i.e. using Lemma 1, is such that the state trajectory diverges if no fault isolation is performed (see red line in Fig. 10). Also, as expected, in the case where the state trajectory converges to zero, the control inputs are inside the saturation limits, as shown in Fig. 11.

\section{Conclusions}

In this paper, the problem of FTC of unstable systems subject to actuator saturation and fault isolation delay has been considered. The adopted solution relies on virtual actuators, a fault-hiding active FTC strategy that reconfigures the faulty plant instead of the controller. Some conditions have been obtained for designing the virtual actuators in such a way that it is guaranteed that, if at the fault isolation time the closed-loop system state is inside a region defined by a value of the Lyapunov function, the state trajectory will converge to zero despite the fault and, moreover, the inputs will not saturate at any time. Afterwards, the problem of delays in the fault isolation has been considered by showing that an estimation of the allowed fault isolation delay can be obtained by analyzing the Lyapunov function using the notion of LMI regions. Moreover, the nominal controller can be designed so as to maximize the allowed fault isolation delay.

A numerical example has shown the effectiveness of the proposed strategy. In particular, it has been demonstrated that the proposed design strategy 


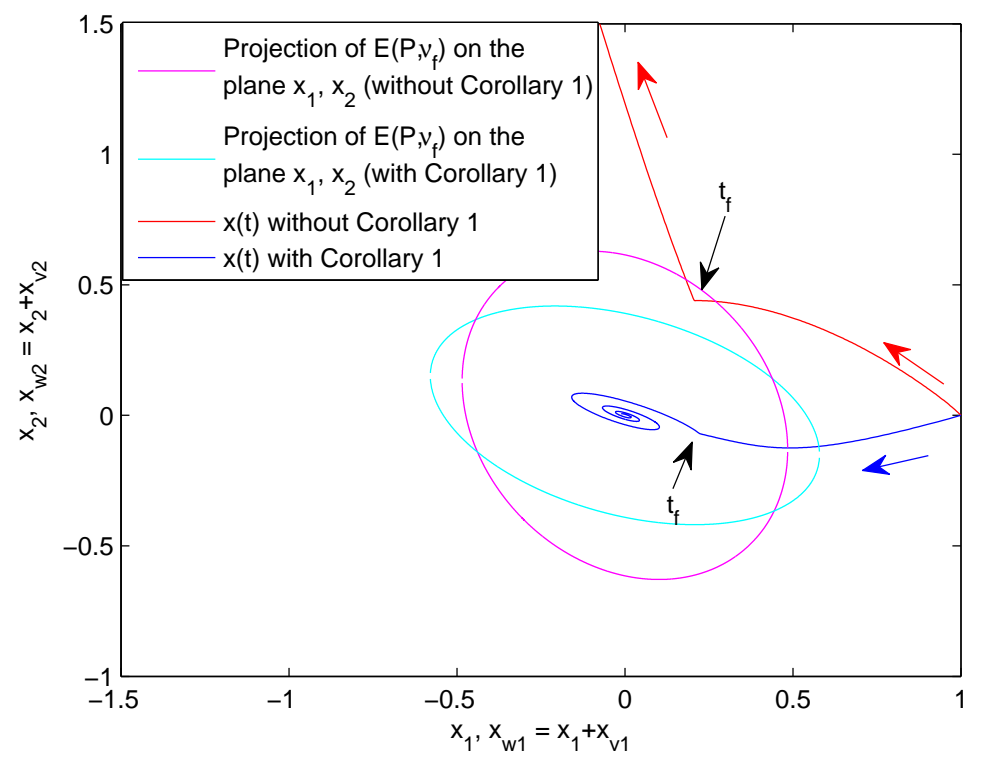

Figure 10: Comparison between the state trajectories obtained with the controllers designed using Lemma 1 and Corollary $1\left(\lambda=0, t_{f}=2 s\right)$, respectively, when no fault isolation is performed during the simulation. 


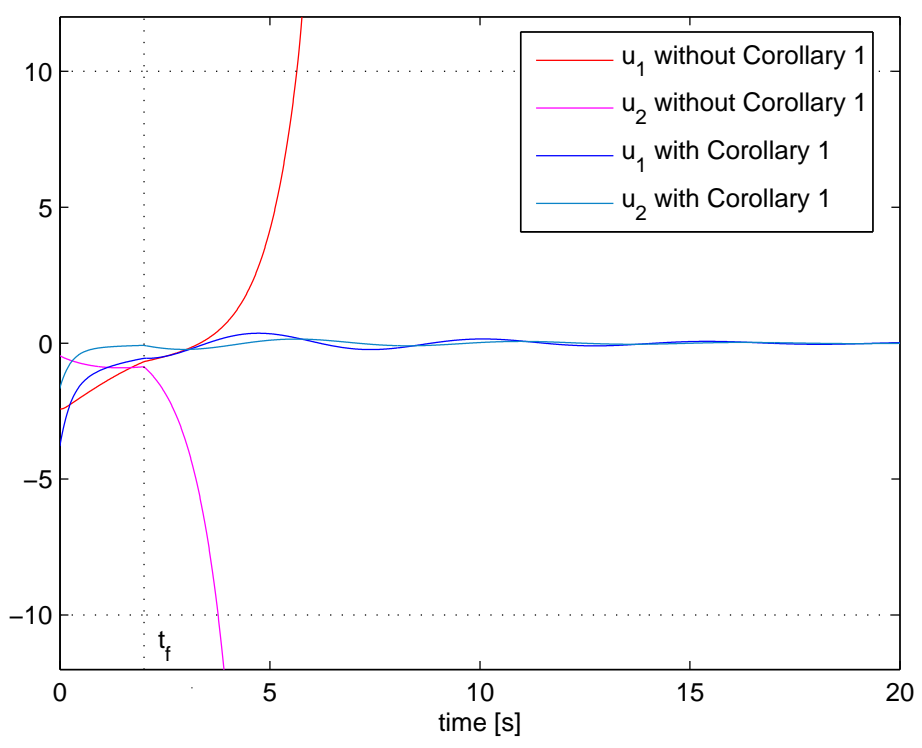

Figure 11: Control inputs with and without applying Corollary $1, t_{f}=2 s$, and no fault isolation during the simulation $\left(t_{I}>20 s\right)$.

enhances the performances of the control system against fault isolation delay. As a special result of the design conditions, it has been obtained a controller that is robust against the considered fault, such that no fault isolation is needed for the system to keep its stability, and for the state trajectory to converge asymptotically to zero under fault occurrence.

\section{Acknowledgments}

This work has been funded by the Spanish Ministry of Science and Technology through the projects CICYT SHERECS (ref. DPI2011-26243) and CICYT ECOCIS (ref. DPI2013-48243-C2-1-R), by AGAUR through the contract FI-DGR 2014 (ref. 2014FI_B1 00172) and by the DGR of Generalitat de Catalunya (SAC group Ref. 2014/SGR/374).

\section{References}

[1] S. Tarbouriech, M. Turner, Anti-windup design: an overview of some recent advances and open problems, IET Control Theory and Applications vol. 3 (2009) pp. 1-19. 
[2] S. Tarbouriech, G. Garcia, J. M. Gomes da Silva Jr., I. Queinnec, Stability and stabilization of linear systems with saturating actuators, Springer-Verlag, London, 2011.

[3] E. F. Mulder, M. V. Kothare, M. Morari, Multivariable anti-windup controller synthesis using linear matrix inequalities, Automatica vol. 37 (2001) pp. 1407-1416.

[4] G. Grimm, J. Hatfield, I. Postlethwaite, A. R. Teel, M. C. Turner, L. Zaccarian, Antiwindup for stable linear systems with input saturation: an LMI-based synthesis, IEEE Transactions on Automatic Control vol. 48 (2003) pp. 1509-1525.

[5] J. M. Gomes da Silva, S. Tarbouriech, Local stabilization of discretetime linear systems with saturating controls: an LMI-based approach, IEEE Transactions on Automatic Control vol. 46 (2001) pp. 119-125.

[6] H. J. Sussmann, E. D. Sontag, Y. Yang, A general result on the stabilisation of linear systems using bounded controls, IEEE Transactions on Automatic Control vol. 39 (1994) pp. 2411-2425.

[7] M. Blanke, M. Kinnaert, J. Lunze, M. Staroswiecki, Diagnosis and FaultTolerant Control, Springer-Verlag Berlin Heidelberg, 2006.

[8] H. Noura, D. Theilliol, J.-C. Ponsart, A. Chamseddine, Fault-tolerant control systems: Design and Practical Applications, Springer-Verlag London Limited, 2009.

[9] J. Jiang, X. Yu, Fault-tolerant control systems: a comparative study between active and passive approaches, Annual Reviews in Control vol. 36 (2012) pp. 60-72.

[10] Y. Zhang, J. Jiang, Bibliographical review on reconfigurable faulttolerant control systems, Annual reviews in control vol. 32 (2008) pp. 229-252.

[11] M. Benosman, A survey of some recent results on nonlinear fault tolerant control, Mathematical Problems in Engineering (2010) pp. 1-25.

[12] T. Steffen, Control reconfiguration of dynamical systems: Linear approaches and structural tests, volume 230 of Lecture Notes in Control and Information Sciences, Springer, 2005. 
[13] J. Lunze, T. Steffen, Control reconfiguration after actuator failures using disturbance decoupling methods, IEEE Transactions on Automatic Control vol. 51 (2006) pp. 1590-1601.

[14] D. Rotondo, F. Nejjari, V. Puig, A virtual actuator and sensor approach for fault tolerant control of LPV systems, Journal of Process Control vol. 24 (2014) pp. 203-222.

[15] L. Dziekan, M. Witczak, J. Korbicz, Active fault-tolerant control design for Takagi-Sugeno fuzzy systems, Bulletin of the Polish Academy of Sciences vol. 59 (2011) pp. 93-102.

[16] J. H. Richter, W. P. M. H. Heemels, N. van de Wouw, J. Lunze, Reconfigurable control of piecewise affine systems with actuator and sensor faults: stability and tracking, Automatica vol. 47 (2011) pp. 678-691.

[17] M. J. Khosrowjerdi, S. Barzegary, Fault tolerant control using virtual actuator for continuous-time Lipschitz nonlinear systems, International Journal of Robust and Nonlinear Control vol. 24 (2013) 2597-2607.

[18] J. H. Richter, Reconfigurable control of nonlinear dynamical systems A fault-hiding approach, Springer-Verlag, Heidelberg, 2011.

[19] J. Blesa, D. Rotondo, V. Puig, F. Nejjari, FDI and FTC of wind turbines using the interval observer approach and virtual actuators/sensors, Control Engineering Practice vol. 24 (2014) pp. 138-155.

[20] J. H. Fan, Y. M. Zhang, Z. Q. Zheng, Robust fault-tolerant control against time-varying actuator faults and saturation, IET Control Theory and Applications vol. 6 (2012) pp. 2198-2208.

[21] M. Benosman, K.-Y. Lum, Application of absolute stability theory to robust control against loss of effectiveness, IET Control Theory and Applications vol. 2 (2009) pp. 772-788.

[22] Z. Zuo, D. Ho, Y. Wang, Fault tolerant control for singular systems with actuator saturation and nonlinear perturbation, Automatica vol. 46 (2010) pp. 569-576. 
[23] B. Xiao, Q. L. Hu, Y. M. Zhang, Adaptive sliding mode fault tolerant attitude tracking control for flexible spacecraft under actuator saturation, IEEE Transactions on Control Systems Technology vol. 20 (2012) pp. $1605-1612$.

[24] P. Mhaskar, C. McFall, A. Gani, P. D. Christofides, J. F. Davis, Isolation and handling of actuator faults in nonlinear systems, Automatica vol. 44 (2008) pp. 53-62.

[25] J. Jiang, Y. M. Zhang, Accepting performance degradation in faulttolerant control system design, IEEE Transactions on Control Systems Technology vol. 14 (2006) pp. 284-292.

[26] Y. M. Zhang, J. Jiang, D. Theilliol, Incorporating performance degradation in fault tolerant control system design with multiple actuator failures, International Journal of Control vol. 6 (2008) pp. 327-338.

[27] D. Theilliol, C. Join, Y. M. Zhang, Actuator fault tolerant control design based on a reconfigurable reference input, International Journal of Applied Mathematics and Computer Science vol. 18 (2008) pp. 553560 .

[28] M. Benosman, K.-Y. Lum, Online references reshaping and control reallocation for nonlinear fault tolerant control, IEEE Transactions on Control Systems Technology vol. 17 (2009) pp. 366-379.

[29] J. Stoustrup, H. Niemann, Fault tolerant control for unstable systems: a linear time varying approach, in: Proceedings of the 2004 American Control Conference, pp. 1794-1798.

[30] Z. Weng, R. Patton, P. Cui, Active fault-tolerant control for unstable systems, in: Proceedings of the 4th IFAC Symposium on Mechatronic Systems, pp. 79-84.

[31] Q. Hu, B. Xiao, M. I. Friswell, Robust fault-tolerant control for spacecraft attitude stabilisation subject to input saturation, IET Control Theory and Applications vol. 5 (2011) pp. 271-282.

[32] F. Wu, B. Lu, Anti-windup control design for exponentially unstable LTI systems with actuator saturation, Systems and Control Letters vol. 52 (2004) pp. 305-322. 
[33] T. Hu, Z. Lin, B. M. Chen, An analysis and design method for linear systems subject to actuator saturation and disturbance, Automatica vol. 38 (2002) pp. 351-359.

[34] F. Blanchini, Set invariance in control, Automatica vol. 35 (1999) pp. $1747-1767$.

[35] T. Iwasaki, M. Fu, Regional $H_{2}$ performance synthesis, in: V. Kapila, K. M. Grigoriadis (Eds.), Actuator Saturation Control, 2002.

[36] R. Goebel, T. Hu, A. R. Teel, Dual matrix inequalities in stability and performance analysis of linear differential/difference inclusions, Current Trends in Nonlinear Systems and Control Systems and Control: Foundations and Applications (2006) pp. 103-122.

[37] T. Nguyen, F. Jabbari, Output feedback controllers for disturbance attenuation with actuator amplitude and rate saturation, Automatica vol. 36 (2000) pp. 1339-1346.

[38] M. Chilali, P. Gahinet, P. Apkarian, Robust pole placement in LMI regions, IEEE Transactions on Automatic Control vol. 44 (1999) pp. $2257-2270$.

[39] A. R. Conn, K. Scheinberg, L. N. Vicente, Introduction to derivative-free optimization, MOS-SIAM Series on Optimization, SIAM, Philadelphia, 2009 .

[40] T. Coleman, M. A. Branch, A. Grace, Optimization Toolbox User's Guide, The Mathworks, Inc., Natick, MA, 1999.

[41] J. Löfberg, YALMIP: A toolbox for modeling and optimization in MATLAB, in: Proceedings of the CACSD Conference, Taipei, Taiwan, 2004, pp. pp. $284-289$.

[42] J. F. Sturm, Using SeDuMi 1.02, a MATLAB toolbox for optimization over symmetric cones, Optimization methods and software vol. 11-12 (1999) pp. 625-653. 